\title{
CONJUGACY SEPARABILITY OF CERTAIN FREE PRODUCTS WITH AMALGAMATION
}

BY

\author{
PETER F. STEBE
}

\begin{abstract}
Let $G$ be a group. An element $g$ of $G$ is called conjugacy distinguished or c.d. in $G$ if and only if given any element $h$ of $G$ either $h$ is conjugate to $g$ or there is a homomorphism $\xi$ from $G$ onto a finite group such that $\xi(h)$ and $\xi(g)$ are not conjugate in $\xi(G)$. Following A. Mostowski, a group $G$ is conjugacy separable or c.s. if and only if every element of $G$ is c.d. in $G$. In this paper we prove that every element conjugate to a cyclically reduced element of length greater than 1 in the free product of two free groups with a cyclic amalgamated subgroup is c.d. We also prove that a group formed by adding a root of an element to a free group is c.s.
\end{abstract}

In [4], A. Mostowski defined conjugacy separable groups and showed that the conjugacy problem is soluble for conjugacy separable groups. S. Lipschutz [1] has solved the conjugacy problem for the free products of free groups with cyclic amalgamated subgroups.

In this paper the problem of conjugacy separability of free products of free groups with a cyclic amalgamated subgroup is considered. It is shown that every element conjugate to a cyclically reduced element of length greater than 1 in the free product of two free groups with a cyclic amalgamated subgroup is c.d. Also, it is shown that a group formed from a free group $F$ by adding a new generator $x$ and a single relation $x^{n}=g$ for some $g \in F$ is a conjugacy separable group.

A general reference for theorems in infinite group theory is the book by $\mathrm{W}$. Magnus, A. Karrass and D. Solitar [3]. References to this book are given as M.K.S. followed by the page number or the number of the theorem or corollary cited.

The proof that a cyclically reduced element of length greater than one in the free product of two free groups with a cyclic amalgamated subgroup is conjugacy distinguished depends on certain properties of free groups. The set of lemmas to follow explains these properties.

LEMMA 1. Let $F$ be a free group. Let $g$ be an element of $F$ and let $u$ be an integer. If $g \neq 1$, there is a homomorphism $\chi$ from $G$ onto a finite group such that $\chi(g)$ has order $u$. If $u$ is a power of a prime $q, \chi$ may be chosen so that $\chi(G)$ is a q-group.

Proof. According to a theorem of W. Magnus, the intersection of the groups $F^{k}$ of the lower central series of $F$ is the identity. Since $g \neq 1$, there is an index $n$

Received by the editors August 20, 1970.

AMS 1970 subject classifications. Primary 20E25, 20E30.

Key words and phrases. Group, conjugacy problem, conjugacy separable group, free product with amalgamation.

Copyright (C) 1971, American Mathematical Society 
such that $g$ is an element of $F^{n-1}$ but $g$ is not an element of $F^{n}$. Let $N$ be the subgroup of $F$ generated by $g^{u}$ and $F^{n} . N$ is normal in $F$ since $F^{n}$ is normal in $F$ and the image of $g$ in $F / F^{n}$ is central. If $g^{v}$ is an element of $N, g^{v}=g^{u k} f$, where $f$ is an element of $F^{n}$. Since $F / F^{n}$ is torsion free, $g^{v}$ is an element of $N$ only if $u$ divides $v$. Thus $g$ has order $u$ modulo $N$. Since $N \supset F^{n}, F / N$ is nilpotent. Let $\eta$ be the natural homomorphism from $F$ onto $F / N$. Then $\eta(g)$ has order $u$ and $\eta(F)$ is nilpotent.

By a theorem of $\mathrm{K}$. Hirsch, $\eta(F)$ is residually finite. Let $M$ be a normal subgroup of finite index in $\eta(F)$ not containing $g, g^{2}, \ldots, g^{u-1}$. Let $\xi$ be the natural homomorphism from $\eta(F)$ onto $\eta(F) / M$. Then $\xi \eta(F)$ is finite and $\xi \eta(g)$ has order $u$.

Now suppose $u=q^{e}$ for $q$ a prime. The group $\xi \eta(F)$ is nilpotent since it is an image group of the group $F / N$. Since $\xi \eta(F)$ is finite, it is the direct product of its Sylow $p$-subgroups. Since $\xi \eta(g)$ has order $q^{e}$, there is a direct factor $Q$ of $\xi \eta(F)$ such that $\xi \eta(g) \in Q$ and $Q$ is a finite $q$ group. There is a homomorphism $\delta$ from $\xi \eta(F)$ onto $Q$, such that $\delta$ restricted to $Q$ is the identity. Thus $\delta \xi \eta(F)$ is a finite $q$-group and $\delta \xi \eta(g)$ has order $q^{e}$.

The proof of Lemma 1 was suggested by D. S. Passman.

LEMMA 2. Let $a_{1}, \ldots, a_{k}$ and $b$ be nonidentity elements of a free group $F$. Let $p$ be a given prime number. If $a_{i} \neq b^{z}$ for each $i$ and all integers $z$, there is a normal subgroup $N$ of finite index in $F$ such that $a_{i} \not \equiv b^{z} \bmod N$ for each $i$ and all integers $z$ and the order of $b$ modulo $N$ is a power of $p$.

Proof. Suppose there is an $a_{i}$ such that $\left(a_{i}, b\right) \neq 1$. For each $i$ such that $\left(a_{i}, b\right) \neq 1$ let $M_{i}$ be a normal subgroup of index a power of $p$ in $F$ such that $\left(a_{i}, b\right) \notin M_{i}$. Let $M$ be the intersection of all the $M_{i}$. The group $F / M$ is a $p$-group. If all $a_{i}$ commute with $b$, let $M$ be a normal subgroup of index a power of $p$ in $F$ such that $b \notin M$. Let the order of $b$ modulo $M$ be $p^{e}$.

The element $b$ and all the $a_{i}$ that commute with $b$ generate a cyclic subgroup $C$ of $F$. Let $f$ generate $C$. Thus $b=f^{s}, a_{i}=f^{r_{i}}$ for each $a_{i}$ commuting with $b$, and $s$ divides no $r_{i}$. Let $R$ be a normal subgroup of finite index in $F$ such that $f$ has order $s p^{e}$ modulo $R$.

Let $N=M \cap R$. The element $b$ has order $p^{e}$ modulo each of $M$ and $R$ so $b$ has order $p^{e}$ modulo $N$. If $a_{i}$ commutes with $b, a_{i} \equiv b^{z} \bmod N$ implies $a_{i} \equiv b^{z} \bmod R$ and this congruence implies that $s$ divides $r_{i}$, contrary to hypothesis. If $\left(a_{i}, b\right) \neq 1$ then $\left(a_{i}, b\right) \not \equiv 1 \bmod N$ so $a_{i} \not \equiv b^{z} \bmod N$. Thus, $a_{i} \not \equiv b^{z} \bmod N$ for all $i$ and each $z$.

LEMMA 3. Let $F$ be a finitely generated free group. Let $a, b$ and $c$ be elements of $F$. If the equation $a=b^{n} c^{m}$ has no solution for integral $n$ and $m$, there is a normal subgroup $N$ of finite index in $F$ such that the congruence $a \equiv b^{n} c^{m} \bmod N$ has no integral solutions.

Proof. The proof is divided into several cases.

Case 1. The elements $b$ and $c$ commute. In this case $b$ and $c$ generate a free cyclic subgroup of $F$. Let $f$ generate the cyclic subgroup generated by $a$ and $b$. The 
equation $a=b^{n} c^{m}$ is equivalent to the equation $a=f^{n}$. By Lemma 2, there is a normal subgroup $N$ of finite index in $F$ such that $a \neq f^{n} \bmod N$ for all integers $n$. Thus $a \not \equiv b^{n} c^{m} \bmod N$ for all integers $n, m$.

Case 2. Assume that the elements $b$ and $c$ do not commute. In this case the subgroup generated by $b$ and $c$ is free of rank 2 , and hence is freely generated by $b$ and $c$. Let $H$ be the subgroup generated by $b$ and $c$. According to a theorem of A. Karrass and D. Solitar [2], $H$ is a free factor of a subgroup $M$ of finite index in $F$. Since $M$ is of finite index in a finitely generated free group, $M$ is finitely generated. Since $b$ and $c$ freely generate a free factor of $M$, we may take $x_{1}=b, x_{2}=c, x_{3}, \ldots, x_{n}$ to be the free generators of $M$.

Suppose $a$ is an element of $M$. Let $w$ be the reduced word in the generators $x_{i}$ of $M$ representing $a$. Let $v$ be an integer greater than any exponent in the expression $w=x_{n_{1}}^{e_{1}} \cdots x_{n_{k}}^{e_{k}}$ with $n_{i} \neq n_{i+1}$. Let $\xi$ be the homomorphism from $M$ onto $G=\left(a_{1}, \ldots, a_{n} ; a_{i}^{v}=1\right)$ defined by assigning $\xi\left(x_{i}\right)=a_{i}$. Now $\xi(a) \neq \xi(b)^{s} \xi(c)^{t}$ for all integers $s$ and $t$, since the image of $a$ in $G$ is represented by $a_{n_{1}}^{e_{1}} \cdots a_{n_{k}}^{e_{k}}=w$, and $w$ is a reduced word in $G$. $G$ is the free product of the cyclic groups of order $v$ generated by the $a_{i}$. Now $G$ is residually finite since it is the free product of a set of finite groups. $G$ contains only finitely many elements $\xi(b)^{s} \xi(c)^{t}$. There is a homomorphism $\eta$ from $\xi(M)$ onto a finite group so that $\eta \xi(a) \neq \eta \xi(b)^{s} \eta \xi(c)^{t}$. Let $U$ be the kernel of $\eta \xi$.

If $a$ is not an element of $M$, let $U=M$. In either case, $U$ is of finite index in $M$ and hence $F$. Let $N$ be the intersection of all the conjugates of $U . N$ is a normal subgroup of finite index in $F$. Since $a^{-1} b^{n} c^{m}$ is not an element of $U$ for all $n, m$ and $U \supset N, a \not \equiv b^{n} c^{m} \bmod N$ for all $n$ and all $m$.

LeMma 4. Let $F$ be a free group. Let $g, h$ be elements of $F$. If $(g, h) \neq 1$, then $\left(h^{-1} g h, g\right) \neq 1$.

Proof. Let $S$ be the subgroup of $F$ generated by $g$ and $h . S$ is free of rank 1 or 2 . If $S$ has rank 1 , it is abelian and $(g, h)=1$, contrary to hypothesis. If $S$ is free of rank 2 , it is freely generated by $g$ and $h$, so that $\left(h^{-1} g h, g\right) \neq 1$. Q.E.D.

Lemma 5. Let $F_{1}$ and $F_{2}$ be free groups. For each $i$, let $N_{i}$ be a normal subgroup of finite index in $F_{i}$. For each $i$, let $g_{i}$ be a nonidentity element of $F_{i}$. If $g_{i}$ has order $n_{i}$ modulo $N_{i}$, there is a normal subgroup $M_{i}$ of finite index in $F_{i}$ such that $N_{i}$ contains $M_{i}$ and $g_{i}$ has order $n_{1} n_{2}$ modulo $M_{i}$.

Proof. Let $U_{i}$ be a normal subgroup of finite index in $F_{i}$ such that $g_{i}$ has order $n_{1} n_{2}$ modulo $U_{i}$. Let $M_{i}=N_{i} \cap U_{i}$. Since $g_{i}^{n_{1} n_{2}}$ is an element of $N_{i}, g_{i}$ has order $n_{1} n_{2}$ modulo $M_{i}$.

LEMMA 6. Let $G$ be a group. Let $g$ and $h$ be elements of $G$. Let $p$ be a prime. Let $g$ have order $p^{e}$ in $G$. If $\left(g, h^{-1} g h\right) \neq 1$ and $h^{-1} g^{r} h=g^{s}$, then $p$ divides $r$ and s.

Proof. If $r$ were relatively prime to the order of $g$, we would have $h^{-1} g h$ $=\left(h^{-1} g^{\top} h\right)^{v}=g^{v s}$ for some integer $v$, so that $h^{-1} g h$ would commute with $g$. Thus $p$ 
divides $r$. If $s$ were relatively prime to the order of $g$, we would have $g=g^{s v}$ $=\left(h^{-1} g^{r} h\right)^{v}=\left(h^{-1} g h\right)^{r v}$ for some integer $v$, so that $h^{-1} g h$ would commute with $g$. Thus $p$ divides $s$.

LEMMA 7. Let $G$ be a group. Let $N_{i}$ be a normal subgroup of $G$ for $i=1, \ldots, k$ : Let $b$ be an element of $G$. Let $p$ be a prime number. If the order of $b$ modulo $N_{i}$ is $a$ power of $p$ for each $i, b$ has order a power of $p$ modulo the intersection of the $N_{i}$.

Proof. Since $k$ is finite, we need only the case of two $N_{i}$. Let $b$ have order $p^{e_{1}}$ modulo $N_{1}$ and order $p^{e_{2}}$ modulo $N_{2}$. Assume without loss of generality that $e_{1}$ is greater than or equal to $e_{2}$. Now $b^{p_{1}}$ is an element of both $N_{1}$ and $N_{2}$ and hence of $N_{1} \cap N_{2}$. Thus the order of $b$ modulo $N_{1} \cap N_{2}$ divides $p^{e_{1}}$ and so is a power of $p$.

The next lemma is about the free product of any two groups with a cyclic amalgamated subgroup.

Lemma 8. Let $G$ be the free product of two groups $A$ and $B$ with a cyclic amalgamated subgroup $C$ generated by an element $c$. Let $g$ and $h$ be elements of $G$. Let $g=t_{1} \cdots t_{n}$ and $h=s_{1} \cdots s_{m}$, where each $t_{i}$ is in $A$ or $B$, each $s_{i}$ is in $A$ or $B$, consecutive $t_{i}$ are in different factors of $G$ and consecutive $s_{i}$ are in different factors of $G$. The equation $c^{-z} h c^{z}=g$ is valid for an integer $z$ if and only if there exist integers $u_{0}, \ldots, u_{m}$ such that $t_{i}^{-1} c^{u_{i-1} s_{i}}=c^{u_{i}}$ for $i=1, \ldots, m$ and $u_{0}=u_{m}$.

Proof. If the equations have a solution, clearly $g=c^{u_{0}} h c^{-u_{0}}$. Suppose $g=c^{u_{0}} h c^{-u_{0}}$. Then $t_{1} \cdots t_{n}=c^{u_{0}} s_{1} \cdots s_{m} c^{-u_{0}}$. The left-hand side of the equation has syllable length $n$ while the right-hand side of the equation has syllable length $m$ since $c$ is in the amalgamated subgroup. Thus $n=m$. Also

$$
t_{2} \cdots t_{n}=t_{1}^{-1} c^{u} 0 s_{1} s_{2} \cdots s_{n} c^{-u_{0}} .
$$

The left-hand side of the equation has syllable length $n-1$ so that $t_{1}^{-1} c^{u_{0} s_{1}}$ must be in the same factor of $G$ as $s_{2}$. This is possible only if $t_{1}^{-1} c^{u_{0}} s_{1}$ is in the amalgamated subgroup. Thus $t_{1}^{-1} c^{u_{0}} s_{1}=c^{u_{1}}$ for $u_{1}$ an integer. The process can clearly be continued, so that an induction based on this process will prove the lemma.

In the statements and proofs of Lemmas 9 through 16 we make the following conventions. Let $F_{1}$ and $F_{2}$ be finitely generated free groups. Let $c_{i}$ be an element of $F_{i}, i=1,2$. Let $G$ be the free product of the $F_{i}$ with the cyclic subgroups generated by the $c_{i}$ amalgamated. Let $c$ be the generator of the amalgamated subgroup of $G$.

LEMMA 9. Let $N_{1}$ and $N_{2}$ be normal subgroups of $F_{1}$ and $F_{2}$ respectively. If the order of $c_{1}$ modulo $N_{1}$ equals the order of $c_{2}$ modulo $N_{2}$, there is a homomorphism $\xi$ from $G$ onto the free product of $F_{1} / N_{1}$ and $F_{2} / N_{2}$ with the images of $c_{1}$ and $c_{2}$ amalgamated. The homomorphism $\xi$ acts as the natural homomorphism from $F_{i}$ onto $F_{i} / N_{i}$.

Proof. Lemma 9 is trivial.

LEMMA 10. Let $F_{i} \supset N_{i} \supset M_{i}$ where $N_{i}$ and $M_{i}$ are normal in $F_{i}$ for $i=1,2$. Let the order of $c_{1} \bmod N_{1}$ equal the order of $c_{2} \bmod N_{2}$ and let the order of $c_{1} \bmod M_{1}$ 
equal the order of $c_{2} \bmod M_{2}$. If $\alpha$ is the homomorphism constructed as in Lemma 9 with the $N_{i}$ and $\beta$ is the homomorphism constructed as in Lemma 9 with the $M_{i}$ then the kernel of $\alpha$ contains the kernel of $\beta$.

Proof. Clearly $\alpha(G)$ is a factor group of $\beta(G)$. Thus the kernel of $\alpha$ contains the kernel of $\beta$.

LEMMA 11. Let $g$ and $h$ be elements of $G$. Let $g=t_{1} \cdots t_{n}, h=s_{1} \cdots s_{m}$ be expressions for $g$ and $h$ in terms of syllables $t_{i}, s_{i}$, where consecutive $t_{i}$ and consecutive $s_{i}$ are in different factors of $G$. Let $n>1$. If $m \neq n$ or one of the equations $t_{i}^{-1} c^{u} s_{i}=c^{v}$ has no integral solution $u, v$, there is a homomorphism $\xi$ corresponding to normal subgroups of the $F_{i}$ as in Lemma 9 such that each $\xi\left(F_{i}\right)$ is finite and $\xi(c)^{-z} \xi(g) \xi(c)^{z} \neq \xi(h)$ for all integers $z$.

Proof. First we consider the case $n=m$. Suppose $t_{i}^{-1} c^{u} s_{i}=c^{v}$ has no solutions and that $t_{i}$ and $s_{i}$ are elements of the same factor of $G$. Without loss of generality, let the factor of $G$ be $F_{1}$. Let $N_{1}$ be a normal subgroup of finite index in $F_{1}$ such that

(1) $t_{i}^{-1} c^{u} s_{i} c^{-v} \notin N_{1}$ for all integers $u, v$.

(2) If $t_{j} \in F_{1}, t_{j}^{-1} c^{z} \notin N_{1}$ for all integers $z$.

(3) If $s_{j} \in F_{1}, s_{j} c^{z} \notin N_{1}$ for all integers $z$.

The subgroup $N_{1}$ is the intersection of subgroups provided by Lemmas 2 and 3 . Let $N_{2}$ be a normal subgroup of finite index in $F_{2}$ such that

(1) If $t_{j} \in F_{2}, t_{j} c^{z} \notin N_{2}$ for all integers $z$.

(2) If $s_{j} \in F_{2}, s_{j} c^{z} \notin N_{2}$ for all integers $z$.

The subgroup $N_{2}$ is the intersection of normal subgroups provided by Lemma 2 .

If $t_{i}^{-1} c^{u} s_{i}=c^{v}$ has no solution for $t_{i}$ and $s_{i}$ in different factors of $G$, we omit the property (1) from the properties of $N_{1}$.

Let $N_{1} \supset M_{1}, N_{2} \supset M_{2}$, where $M_{1}$ and $M_{2}$ are normal subgroups of finite index in $F_{1}$ and $F_{2}$ respectively such that the order of $c_{1} \bmod M_{1}$ equals the order of $c_{2} \bmod M_{2}$. Let $\xi$ be the homomorphism of $G$ corresponding to $M_{1}$ and $M_{2}$ according to the construction of Lemma 9.

Suppose there is an integer $z$ such that $\xi(c)^{-z} \xi(g) \xi(c)^{z}=\xi(h)$. Then by Lemma 8 there are integers $u_{0}, \ldots, u_{n}$ such that

$$
\xi\left(t_{i}\right)^{-1} \xi(c)^{u_{i-1}} \xi\left(s_{i}\right)=\xi(c)^{u_{i}}, \quad u_{0}=u_{n},
$$

for $\xi\left(t_{i}\right)$ and $\xi\left(s_{i}\right)$ are the syllables of $\xi(g)$ and $\xi(h)$. Thus

$$
\xi\left(t_{i}^{-1} c^{u_{i-1}} s_{i} c^{-u_{i}}\right)=1 .
$$

But this is impossible, so the result follows in the case $n=m$.

If $n \neq m$, choose $N_{i}$ to be a normal subgroup of finite index in $F_{i}$ such that

(1) If $m=1$ and $s_{1} \in F_{i}$, then $s_{1} \notin N_{i}$.

(2) If $m \neq 1$ and $s_{j} \in F_{i}$, then $s_{j} c^{-z} \notin N_{i}$ for all $z$.

(3) If $t_{j} \in F_{i}$, then $t_{j} c^{-z} \notin N_{i}$ for all $z$. 
Let the subgroups $M_{i}$ and the homomorphism $\xi$ be constructed as above. The syllable length of $\xi(g)$ is $n$. The syllable length of $\xi(h)$ is $m$. Since $m \neq n$, Lemma 8 implies that $\xi(c)^{-z} \xi(h) \xi(c)^{z} \neq \xi(g)$.

LEMMA 12. Let $g=t_{1} \cdots t_{n}, h=s_{1} \cdots s_{n}$ where $n>1$ and the $s_{i}$ and $t_{i}$ are syllables of $g$ and $h$ as in Lemma 11. Let $\left(t_{i}, c\right) \neq 1$ for all $i$ and let each equation $t_{i}^{-1} c^{u_{i-1}} s_{i}=c^{v_{i}}$ have an integral solution. If $c^{-z} h c^{z} \neq g$ for all integers $z$ there is a homomorphism $\xi$ as described in Lemma 9 such that each $\xi\left(F_{i}\right)$ is finite and $\xi\left(c^{-z} h c^{z}\right) \neq \xi(g)$ for all $z$.

Proof. According to Lemma $4,\left(t_{i}^{-1} c t_{i}, c\right) \neq 1$ for all $i$. Let $p$ be a prime number dividing no nonzero difference $u_{1}-v_{n}, u_{2}-v_{1}, \ldots, u_{n}-v_{n-1}$. Let $N_{i}$ be a normal subgroup of index a power of $p$ in $F_{i}$ such that

(1) If $t_{j} \in F_{i}$, then $\left(t_{j}^{-1} c t_{j}, c\right) \notin N_{i}$.

(2) If $s_{j} \in F_{i}$, then $\left(s_{j}^{-1} c s_{j}, c\right) \notin N_{i}$.

The subgroup $N_{i}$ is the intersection of subgroups of index a power of $p$ in $F_{i}$. Lemma 1 is used repeatedly. Since the equation $t_{i}^{-1} c^{u_{i}} S_{i}=c^{v_{i}}$ is valid for integers $u_{i}, v_{i}$, we have $\left(s_{j}, c\right) \neq 1$.

Let $M_{i}$ be a normal subgroup of finite index in $F_{i}$ such that $M_{i}$ is contained in $N_{i}$, the order of $c_{i}$ modulo $M_{i}$ is a power of $p$, and the order of $c_{1}$ modulo $M_{1}$ equals the order of $c_{2}$ modulo $M_{2}$. Let $\xi$ be the homomorphism defined as in Lemma 8 from $G$ onto the free product of $F_{1} / M_{1}$ and $F_{2} / M_{2}$ with the image of $C$ amalgamated.

Suppose $\xi\left(t_{i}\right)^{-1} \xi(c)^{v} \xi\left(s_{i}\right)=\xi(c)^{w}$. Since $\xi\left(t_{i}\right)^{-1} \xi(c)^{u_{i}} \xi\left(s_{i}\right)=\xi(c)^{v_{i}}$, we have $\xi(c)^{w-v_{i}}$ $=\xi\left(t_{i}\right)^{-1} \xi(c)^{v-u_{i}} \xi\left(t_{i}\right)$. Now $\xi\left(\left(t_{i}^{-1} c t_{i}, c\right)\right) \neq 1$ so that $w-v_{i}$ and $v-u_{i}$ are divisible by $p$ according to Lemma 6 . If $\xi(c)^{-z} \xi(h) \xi(c)^{z}=\xi(g)$, there exist integers $w_{0}, \ldots, w_{n}$ such that $\xi\left(t_{i}\right)^{-1} \xi(c)^{w_{i-1}} \xi\left(s_{i}\right)=\xi(c)^{w_{i}}$ with $w_{0}=w_{n}$. Thus $0=w_{n}-w_{0}=u_{1}-v_{n} \bmod p$, $0=w_{n-1}-w_{n-1}=u_{n}-v_{n-1} \bmod p$ etc. Since by choice of $p$ at least one of the differences on the right is incongruent to zero $\bmod p$, we have $\xi(c)^{-z} \xi(h) \xi(c)^{z}$ $\neq \xi(g)$. Q.E.D.

LEMMA 13. Let $g=t_{1} \cdots t_{m}, h=s_{1} \cdots s_{m}$ where $h$ and $g$ are elements of $G$ and the $t_{i}$ and the $s_{i}$ are syllables of $g$ and $h$ respectively. Let $m$ be greater than 1 . Let at least one $\left(t_{i}, c\right) \neq 1$. If each of the equations $t_{i}^{-1} c^{u_{i} s_{i}}=c^{v_{i}}$ has an integral solution but $c^{-z} h c^{z} \neq g$ for all integers $z$, there is a homomorphism $\xi$ as given by Lemma 9 such that each $\xi\left(F_{i}\right)$ is finite and $\xi\left(c^{-z} h c^{z}\right) \neq \xi(g)$ for all integers $z$.

Proof. For each $i$ such that $\left(t_{i}, c\right)=1$ we have $t_{i}^{-1} s_{i}=c^{v_{i}-u_{i}}$. We may set $h=s_{1} \cdots s_{m}$ where $s_{i}=t_{i}$ if $t_{i}$ commutes with $c$, no two adjacent $s_{i}$ are in the same factor of $G$, and no $s_{i}$ is in the amalgamated subgroup. The equations $t_{i}^{-1} c^{u_{i}} s_{i}=c^{v_{i}}$ have solutions $u_{i}, v_{i}$ with $u_{i}=v_{i}$ if $t_{i}$ commutes with $c$. Let $t_{n_{1}}, \ldots, t_{n_{k}}$ be the $t_{i}$ not commuting with $c$ and let $n_{k}>n_{k-1}>\cdots>n_{1}$. If each of the differences

$$
u_{n_{1}}-v_{n_{k}}, \quad u_{n_{2}}-v_{n_{1}}, \ldots, \quad u_{n_{k}}-v_{n_{k-1}}
$$

are zero, there is an integer $z$ such that $c^{-z} h c^{z}=g$. Let $p$ be a prime relatively prime to at least one of the nonzero differences. By Lemma $4,\left(t_{i}, c\right) \neq 1$ implies $\left(t_{i}^{-1} c t_{i}, c\right)$ 
$\neq 1$ since $t_{i}$ is in a factor of $G$. By Lemmas 1 and 2 a normal subgroup $N_{i}$ of $F_{i}$ can be found with the properties:

(1) $N_{i}$ is of finite index in $F_{i}$.

(2) $c_{i}$ has order a power of $p$ modulo $N_{i}$.

(3) If $t_{j} \in F_{i}, t_{j} \not \equiv c_{i}^{z} \bmod N_{i}$ for all $z$.

(4) If $s_{j} \in F_{i}, s_{j} \not \equiv c_{i}^{z} \bmod N_{i}$ for all $z$.

(5) If $t_{j} \in F_{i},\left(t_{j}, c_{i}\right) \neq 1,\left(t_{j}^{-1} c_{i} t_{j}, c_{i}\right) \notin N_{i}$.

(6) If $s_{j} \in F_{i},\left(s_{j}, c_{i}\right) \neq 1,\left(s_{j}^{-1} c_{i} s_{j}, c_{i}\right) \notin N_{i}$.

The subgroups $N_{i}$ are found by intersecting the normal subgroups of index a power of $p$ given by Lemma 1 for properties (5) and (6) with normal subgroups of finite index given by Lemma 2 for properties (3) and (4) and the prime $p$. By Lemma $7, c_{i}$ has order a power of $p$ modulo $N_{i}$. Let $M_{i}$ be a normal subgroup of finite index in $F_{i}$ such that $N_{i}$ contains $M_{i}$ and the order of $c_{1}$ modulo $M_{1}$ equals the order of $c_{2}$ modulo $M_{2}$. By Lemma 5 , the $M_{i}$ can be chosen so that the order of $c_{i}$ modulo $M_{i}$ is a power of $p$. Let $\xi$ be the homomorphism from $G$ onto the free product of the groups $F_{i} / M_{i}$ with the images of the $c_{i}$ amalgamated, as given by Lemma 9 . Let $g$ denote the image $\xi(g)$ of $g \in G$.

Suppose $\left(\boldsymbol{t}_{i}, c\right) \neq 1$. By the properties of the $M_{i}$ we have $\left(\boldsymbol{t}_{i}^{-1} \boldsymbol{c} \boldsymbol{t}_{i}, \boldsymbol{c}\right) \neq 1$. If $\boldsymbol{t}_{\boldsymbol{i}}^{-1} \boldsymbol{c}^{a_{i} \boldsymbol{t}_{i}}$ $=c^{b_{i}}$, then $c^{b_{i}-v_{i}}=\boldsymbol{t}_{i}^{-1} c^{a_{i}-u_{i}} \boldsymbol{t}_{i}$. By Lemma $6, a_{i}-u_{i}$ and $b_{i}-v_{i}$ are divisible by $p$. If $\left(t_{i}, c\right)=1$, then $t_{i}=s_{i}$ so that $a_{i}=b_{i}$.

Now $\boldsymbol{t}_{\boldsymbol{i}}$ and $\boldsymbol{s}_{\boldsymbol{i}}$ are the syllables of $\boldsymbol{g}$ and $\boldsymbol{h}$ respectively, so that $\boldsymbol{c}^{-\boldsymbol{z}} \boldsymbol{h} \boldsymbol{c}^{\boldsymbol{z}}=\boldsymbol{g}$ if and only if the equations $\boldsymbol{t}_{i}^{-1} \boldsymbol{c}^{a_{i}} \boldsymbol{s}_{i}=\boldsymbol{c}^{b_{i}}$ have integral solutions $a_{i}, b_{i}$ with $c^{a_{1}}=c^{b_{n}}$, $c^{a_{2}}=c^{b_{1}}, \ldots, c^{a_{n}}=c^{b_{n-1}}$. Suppose there are such solutions to these equations. The elements of the list $a_{1}-b_{n}, a_{2}-b_{1}, \ldots, a_{n}-b_{n-1}$ are each congruent to zero modulo the order of $c$ and hence congruent to zero modulo $p$. It follows from the last paragraph that $a_{1} \equiv a_{n_{1}}, b_{n} \equiv b_{n_{k}}$ and $a_{1}-b_{n}$ is congruent modulo $p$ to $u_{n_{1}}-v_{n_{k}}$. In general each of the differences $u_{n_{i}}-v_{n_{i-1}}$ is congruent to an element of $a_{1}-b_{n}, \ldots, a_{n}-b_{n-1}$ and at least one of the differences $u_{n_{i}}-v_{n_{i-1}}, u_{n_{1}}-v_{n_{k}}$ is not congruent to zero modulo $p$. Thus $\boldsymbol{c}^{-z} \boldsymbol{h} \boldsymbol{c}^{z} \neq \boldsymbol{g}$ for all $z$.

Lemma 14. Let $g=t_{1} \cdots t_{n}, h=s_{1} \cdots s_{n}$, where $g$ and $h$ are elements of $G$ and the $t_{i}$ and $s_{i}$ are syllables of $g$ and $h$ respectively. Let $m$ be greater than one. Let every $t_{i}$ commute with $c$. If each of the equations $t_{i}{ }^{-1} c^{u_{i}} S_{i}=c^{v_{i}}$ has an integral solution $u_{i}, v_{i}$ but $c^{-z} g c^{z} \neq h$ for all integers $z$, there is a homomorphism $\xi$ as given by Lemma 9 such that each $\xi\left(F_{i}\right)$ is finite and $\xi\left(c^{-z} g c^{z}\right) \neq \xi(h)$ for all integers $z$.

Proof. Let $f=g h^{-1}$. Since $c^{-z} g c^{z} \neq h$ for all $z, f \neq 1$.

Suppose $f$ is in a factor of $G$. Without loss of generality assume $f$ is an element of $F_{1}$. Let $N_{1}$ be a normal subgroup of finite index in $F_{1}$ not containing $f$. Let $N_{2}$ be a normal subgroup of finite index in $F_{2}$ such that the order of $c_{2}$ modulo $N_{2}$ equals the order of $c_{1}$ modulo $N_{1}$. Let $\xi$ be the homomorphism constructed according to Lemma 9 using the $N_{i}$. Clearly $\xi(f) \neq 1$. 
If $f$ is not in a factor of $G$, let $f=u_{1} \cdots u_{k}$ where each $u_{i}$ is in a factor of $G$, adjacent $u_{i}$ are in different factors of $G$, and no $u_{i}$ is in the amalgamated subgroup of $G$. Let $N_{i}$ be a normal subgroup of finite index in $F_{i}$ such that if $u_{j} \notin F_{i}$, then $u_{j} c_{i}^{-z} \notin N_{i}$ for all integers $z$. Let $M_{i}$ be a normal subgroup of finite index in $F_{i}$ such that $N_{i} \supset M_{i}$ and the orders of $c_{i}$ modulo $M_{i}$ are equal. Let $\xi$ be the homomorphism constructed according to Lemma 9 using the $M_{i}$. Clearly $\xi(f) \neq 1$.

Since each $t_{i}$ commutes with $c, g$ commutes with $c$ and hence $\xi(g)$ commutes with $\xi(c)$. Thus $\xi(c)^{-z} \xi(g) \xi(c)^{z}=\xi(h)$ if and only if $\xi(g)=\xi(h)$ or $\xi(f)=1$. Thus the lemma is proven.

LEMMA 15. Let $g$ be a cyclically reduced element of length greater than one in $G$. Let $g=t_{1} \cdots t_{m}$ where $m>1, t_{i}$ is an element of a factor of $G$, consecutive $t_{i}$ are in different factors of $G$, no $t_{i}$ is in the amalgamated subgroup and $t_{1}$ and $t_{m}$ are in different factors of $G$. Let $h$ be a cyclically reduced element of $G$. Let $h=s_{1} \cdots s_{n}$ where $n \geqq 1$, consecutive $s_{i}$ are from different factors of $G$, and if $n>1$, no $s_{i}$ is in the amalgamated subgroup and $s_{1}$ and $s_{n}$ are from different factors of $G$. If for each cyclic permutation $\varphi$ of $1, \ldots, m$ and all integers $z$ we have $c^{-z} t_{\varphi(1)} \cdots t_{\varphi(m)} c^{z} \neq h$, there is a homomorphism $\xi$ from $G$ onto the free product of two finite groups with $a$ cyclic amalgamated subgroup such that $\xi\left(t_{i}\right)$ are the syllables of $\xi(g), \xi\left(s_{i}\right)$ are the syllables of $\xi(h), \xi(g)$ is cyclically reduced, $\xi(h)$ is cyclically reduced, $\xi(c)$ generates the amalgamated subgroup of $\xi(G)$ and $\xi\left(c^{-z} t_{\varphi(1)} \cdots t_{\varphi(m)} c^{z}\right) \neq \xi(h)$ for all integers $z$ and each cyclic permutation $\varphi$ of $1, \ldots, m$.

Proof. By Lemmas 11 through 14 there is for each $\varphi$ a homomorphism $\xi_{\varphi}$ from $G$ onto the free product of $\xi_{\varphi}\left(F_{1}\right)$ and $\xi_{\varphi}\left(F_{2}\right)$ with the images of the $c_{i}$ amalgamated such that $\xi \varphi\left(c^{-z} t_{\varphi(1)} \cdots t_{\varphi(m)} c^{z}\right) \neq \xi_{\varphi}(h)$ for all integers $z$. Let $K_{\varphi}$ be the kernel of $\xi_{\varphi}$. Let $K_{\varphi, i}=K_{i} \cap F_{i}$. Let $K_{i}=\bigcap K_{\varphi, i}$ so that each $K_{i}$ is a normal subgroup of finite index in $F_{i}$. Let $M_{i}$ be a normal subgroup of finite index in $F_{i}$ such that $M_{i} \subset K_{i}$, $t_{j} c^{-z} \notin M_{i}, s_{j} c^{-z} \notin M_{i}$ for all $j$ and all integers $z$ and the orders of $c_{i}$ modulo $M_{i}$ are equal. Let $\xi$ be the homomorphism obtained by Lemma 9 using the $M_{i}$. Let $K$ be the kernel of $\xi$. By Lemma $10, K \subset K_{\varphi}$ for each $\varphi$, so that $\xi$ is the required homomorphism.

LEMMA 16. Let $G$ be the free product of two finite groups with an amalgamated subgroup. If $g$ is a cyclically reduced element of length greater than one in $G, g$ is c.d. in $G$.

Proof. According to B. H. Neumann [5, p. 532], there is a homomorphism $\xi$ from $G$ onto a finite group such that the kernel of $\xi$ meets each factor of $G$ only in the identity. According to a theorem of H. Neumann, M.K.S., Corollary 4.9.2, the kernel of $\xi$ is free. Thus $G$ is a finite extension of a free group. Since $g$ is cyclically reduced and has syllable length greater than one, $g$ is of infinite order in $G$. It follows from a theorem of the author [7] that $g$ is c.d. in $G$. 
THEOREM 1. If $G$ is the free product of two free groups with a cyclic amalgamated subgroup, every element of $G$ conjugate to a cyclically reduced element of length greater than one is c.d. in $G$.

Proof. Clearly we need only consider $g$ cyclically reduced in $G$. Let $h$ be a cyclically reduced element of $G$. Let $g=t_{1} \cdots t_{m}, h=s_{1} \cdots s_{n}$ where $m>1, n \geqq 1$, consecutive $t_{i}$ are elements of different factors of $G$, consecutive $s_{i}$ are in different factors of $G$, no $t_{i}$ is in the amalgamated subgroup of $G, t_{1}$ and $t_{m}$ are from different factors of $G, s_{1}$ and $s_{n}$ are from different factors of $G$ if $n>1$, and no $s_{i}$ is in the amalgamated subgroup if $n>1$. According to a theorem of D. Solitar, M.K.S., Theorem $4.6, g$ is conjugate to $h$ if and only if there is a cyclic permutation $\varphi$ of $1, \ldots, m$ and an integer $z$ such that $c^{-z} t_{\varphi(1)} \cdots t_{\varphi(m)} c^{z}=h$. Since this equation is untrue for all $\varphi$ and $z$, there is, by Lemma 15, a homomorphism $\xi$ from $G$ onto the free product of two finite groups such that $\xi(g)$ is cyclically reduced, $\xi\left(t_{i}\right)$ are the syllables of $\xi(g), \xi(g)$ has syllable length greater than $1, \xi\left(s_{i}\right)$ are the syllables of $\xi(h)$ and for each cyclic permutation $\varphi$ of $1, \ldots, m, \xi\left(c^{-z} t_{\varphi(1)} \cdots t_{\varphi(m)} c^{z}\right) \neq \xi(h)$ for all $z$. Since the quoted theorem of D. Solitar applies to $\xi(G), \xi(g)$ and $\xi(h)$ are not conjugate in $\xi(G)$. By Lemma $16, \xi(g)$ is c.d. in $\xi(G)$ so there is a homomorphism $\chi$ from $\xi(G)$ onto a finite group such that $\chi \xi(g) \nsim \chi \xi(h)$.

If $h$ is not cyclically reduced, let $h^{\prime}=h^{x}$ where $h^{\prime}$ is cyclically reduced. Since $h \approx g, h^{\prime} \nsim g$ so that by the last paragraph there is a homomorphism $\chi \xi$ from $G$ onto a finite group so that $\chi \xi(g) \nsim \chi \xi\left(h^{\prime}\right)$. But then $\chi \xi(g) \nsim \chi \xi(h)$. Thus $g$ is c.d. in $G$.

In the next lemma we consider the group $G$ formed by adding a single relation $x^{n}=g$ to the free product of a free group $F$ and the free cyclic group generated by a generator $x$. We always let $g$ be an element of $F$ and call $G$ the group formed by adding a root of an element to a free group. The notation for $G$ as constructed above is $G=\left(F, x ; x^{n}=g\right)$.

Note that if $F$ is a free group $G=\left(F, x ; x^{n}=g\right)$ is a free product of two free groups with a cyclic amalgamated subgroup. By Theorem 1 , every element of $G$ conjugate to a cyclically reduced element of length greater than one is c.d. in $G$. Thus to prove that $G$ is c.s. we need only consider elements of length one in $G$.

LEMMA 17. Let $F$ be a free group and let $G=\left(F, x ; x^{n}=g\right)$ with $g \in F$. If $g_{1}$ and $g_{2}$ are nonconjugate elements of $F$ or distinct powers of $x$, there is a homomorphism $\xi$ from $G$ onto a finite group such that $\xi\left(g_{1}\right)$ is not conjugate to $\xi\left(g_{2}\right)$ in $\xi(G)$.

Proof. The proof is divided into two cases.

Case 1. Let $g_{1}$ and $g_{2}$ be two nonconjugate elements of $F$. Since $F$ is c.s., there is a normal subgroup $N$ of finite index in $F$ such that $g_{1} \not \equiv h^{-1} g_{2} h \bmod N$ for $h \in F$. Let $g$ have order $m$ modulo $N$ and $\xi$ be the natural homomorphism from $F$ onto $F / N$. Let $\psi$ be the homomorphism from $G$ onto $H=\left(F / N, y ; y^{n}=\xi(g)\right)$ defined as follows: $\psi(u)=\xi(u)$ for $u \in F, \psi\left(x^{r}\right)=y^{r}, \psi(a b)=\psi(a) \cdot \psi(b)$. Now $\psi$ is a homomorphism since $\psi\left(x^{n}\right)=y^{n}=\xi(g)$ and $\psi(g)=\xi(g)$. Let $M$ be the set of matrices 
with entries in the integral group ring $R$ of $F / N$. We set $\varphi(u)=\operatorname{diag}(u, \ldots, u)$ for $u \in F / N$ and $\varphi(y)=\operatorname{diag}(1, \ldots, 1, \xi(g)) \cdot P$ where $P$ is the $n \times n$ permutation matrix corresponding to the cycle $(1,2, \ldots, n)$. Now $\varphi(u)^{-1}=\operatorname{diag}\left(u^{-1}, \ldots, u^{-1}\right)$ for $u \in F / N, \varphi\left(y^{n}\right)=\varphi(\xi(g)), \psi(y)^{-1}=P^{n-1} \operatorname{diag}\left(1, \ldots, 1, \xi(g)^{-1}\right)$, so that the matrices $\psi(H)$ generate a group $U$ in $M$, and $\psi$ is a homomorphism from $H$ onto the group $U$. If $D$ is a diagonal matrix, $D P=P D^{*}$ where $D^{*}$ is a diagonal matrix whose entries are, up to order along the diagonal, the same as those of $D$. Thus if $T$ is an element of $U, T=P^{r} \operatorname{diag}\left(d_{1}, \ldots, d_{n}\right)$ where the $d_{i}$ are elements of $F / N$. Thus $U$ is finite.

Suppose $\varphi \psi\left(g_{1}\right)$ is conjugate to $\varphi \psi\left(g_{2}\right)$ in $U$. Then

$$
\begin{aligned}
& \operatorname{diag}\left(\xi\left(g_{1}\right), \ldots, \xi\left(g_{1}\right)\right) \\
& \quad=\operatorname{diag}\left(d_{1}^{-1}, \ldots, d_{n}^{-1}\right) P^{-t} \operatorname{diag}\left(\xi\left(g_{2}\right), \ldots \xi\left(g_{2}\right)\right) P^{t} \operatorname{diag}\left(d_{1}, \ldots, d_{n}\right) .
\end{aligned}
$$

Now $P^{t}$ commutes with $\operatorname{diag}\left(\xi\left(g_{2}\right), \ldots, \xi\left(g_{2}\right)\right)$ so that one has $\xi\left(g_{1}\right)=d_{i}^{-1} \xi\left(g_{2}\right) d_{i}$ for each $d_{i}, d_{i} \in S / N$. But if $h \in \xi^{-1}\left(d_{1}\right)$ we have $g_{1} \equiv h^{-1} g_{2} h \bmod N$, contrary to hypothesis. Thus $\varphi \psi\left(g_{1}\right)$ is not conjugate to $\varphi \psi\left(g_{2}\right)$ in the finite group $U$.

Case 2. Let $g_{1}=x^{i}$ and $g_{2}=x^{j}$, and let $g_{1}$ not be conjugate to $g_{2}$. One has $i \neq j$. If $g_{1}^{n}=g^{i}$ is not conjugate to $g_{2}^{n}=g^{j}$ in $F$, according to Case 1 , there is a homomorphism $\gamma$ from $G$ onto a finite group such that $\gamma\left(g_{1}^{n}\right)$ is not conjugate to $\gamma\left(g_{2}^{n}\right)$ in $\gamma(G)$. Now $\gamma\left(g_{1}\right)=h^{-1} \gamma\left(g_{2}\right) h$ implies $\gamma\left(g_{1}\right)^{n}=h^{-1} \gamma\left(g_{2}\right)^{n} h$ so $\gamma\left(g_{1}\right)$ and $\gamma\left(g_{2}\right)$ are not conjugate. Let there be an $h$ in $F$ such that $h^{-1} g^{i} h=g^{j}$ for $i \neq j$. The subgroup $S$ generated by $g$ and $h$ must be free of rank $\leqq 2$. If $S$ has rank $2, g$ and $h$ are free generators of $S$ and $i=j=0$, contrary to hypothesis. If $S$ has rank $1, S$ is abelian and $g^{i}=g^{j}$. Since $F$ is torsion free and $i \neq j, g$ is the identity. But if $g$ is the identity, $G$ is a free product of $F$ and the cyclic group of order $n$ generated by $x$. Thus $G$ is c.s. by Theorem 2 of [7] and the result follows.

REMARK. The matrix construction used here is based on a construction in the book by A. Speiser [6]. D. S. Passman has remarked that a representation of $G$ as a wreath product would be sufficient.

TheOREM 2. Let $F$ be a free group. Let $g$ be an element of $F$. If $G=\left(F, x ; x^{n}=g\right)$, then $G$ is c.s.

Proof. Let $f$ be an element of $G$ and let $h$ be an element of $G$ not conjugate to $f$. If either $f$ or $h$ is conjugate to a cyclically reduced element of length greater than one then it is c.d. in $G$ by Theorem 1 so that there is a homomorphism $\xi$ from $G$ onto a finite group with the property $\xi(f) \sim \xi(h)$. Thus we may assume that $f$ and $h$ are conjugate to elements of length one in $G$, and we need only consider $f$ and $h$ cyclically reduced.

If $f$ and $h$ are in the same factor of $G$, Lemma 17 implies that there is a homomorphism $\xi$ from $G$ onto a finite group such that $\xi(f) \approx \xi(h)$.

If $f$ and $h$ are in different factors of $G$, neither is in the amalgamated subgroup. Thus one of $f$ and $h$ is a power of $x$ but not a power of $g$. Let $\xi$ be the homo- 
morphism from $G$ onto the group $\left(y ; y^{n}=1\right)$ determined by the assignments $x \rightarrow y, F \rightarrow 1$. Clearly $\xi$ is a homomorphism from $G$ onto a finite group and $\xi(f) \nsim \xi(h)$.

Thus every element of $G$ is c.d. in $G$ so $G$ is c.s.

\section{REFERENCES}

1. S. Lipshutz, Generalization of Dehn's result on the conjugacy problem, Proc. Amer. Math. Soc. 17 (1966), 759-762. MR 33 \#5706.

2. A. Karrass and D. Solitar, On finitely generated subgroups of a free group, Proc. Amer. Math. Soc. 22 (1969), 209-213. MR 39 \#6961.

3. W. Magnus, A. Karrass and D. Solitar, Combinatorial group theory, Pure and Appl. Math., vol. 13, Interscience, New York, 1966. MR 34 \#7617.

4. A. W. Mostowski, On the decidability of some problems in special classes of groups, Fund. Math. 59 (1966), 123-135. MR 37 \#292.

5. B. H. Neumann, An essay on free products of groups with amalgamations, Philos. Trans. Roy. Soc. London Ser. A 246 (1954), 503-554. MR 16, 10.

6. A. Speiser, Theorie der Gruppen von endlicher Ordnung, 3rd ed., Springer, Berlin, 1936.

7. P. Stebe, A residual property of certain groups, Proc. Amer. Math. Soc. 26 (1970), 37-42.

Institute for Defense ANAlyses,

Princeton, New Jersey 08540 